\title{
Effect of surfactants on the morphology of FeSe films fabricated from a single source precursor by aerosol assisted chemical vapour deposition
}

\author{
RAJA AZADAR HUSSAIN ${ }^{\mathrm{a}}$, AMIN BADSHAH $^{\mathrm{a}, *}$, NAGHMA HAIDER ${ }^{\mathrm{b}}$, \\ MALIK DILSHAD KHAN ${ }^{\mathrm{a}}$ and BHAJAN LAL ${ }^{\mathrm{c}}$ \\ ${ }^{a}$ Department of Chemistry, Quaid-i-Azam University, Islamabad (45320) Pakistan \\ ${ }^{\mathrm{b}}$ Geoscience Advanced Research Laboratories, Geological Survey of Pakistan, Chakshahzad Town, \\ Islamabad Pakistan \\ ${ }^{c}$ Department of Chemistry, Shah Abdul Latif University, Khairpur, Pakistan \\ e-mail: aminbadshah@yahoo.com
}

MS received 21 April 2014; revised 25 August 2014; accepted 3 September 2014

\begin{abstract}
This article presents the fabrication of FeSe thin films from a single source precursor namely (1(2-fluorobenzoyl)-3-(4-ferrocenyl-3-methylphenyl)selenourea (MeP2F)) by aerosol assisted chemical vapour deposition (AACVD). All the films were prepared via similar experimental conditions (temperature, flow rate, concentration, solvent system and reactor type) except the use of three different concentrations of two different surfactants i.e., triton and span. Seven thin films were characterized with PXRD, SEM, AFM, EDS and EDS mapping. The mechanism of the interaction of surfactant with MeP2F was determined with cyclic voltammetry (CV) and UV-Vis spectroscopy.
\end{abstract}

Keywords. Single source precursor; chemical vapour deposition; cyclic voltammetry; UV-Vis spectroscopy.

\section{Introduction}

Conventional applications of the surfactants as clean solubilizers have been updated in recent years and now they are being used as templates for the synthesis of ceramics, polymers and nanomaterials. ${ }^{1}$ The fascinating aspect in the material fabrication using surfactants is the use of their micelle droplets as nanoreactors. The use of surfactants in liquid phase affects the particle size, orientation, morphology, mechanical strength, corrosion, luminescence and photoelectric properties of the deposited materials. ${ }^{2-4}$ However, the effect of surfactants in gas phase, aerosol- assisted chemical vapour deposition (AACVD) has rarely been discussed..$^{5}$ In this study, we have used two surfactants, triton and span, to get a glimpse of the effect of their presence on the morphologies of the FeSe films fabricated from a single source ferrocene-incorporated selenourea precursor (MeP2F) which we have recently reported for biological applications. ${ }^{6-9}$ The only variable parameter during AACVD of MeP2F was the concentration of the surfactants and all other experimental conditions such as temperature, flow rate, concentration of the precursor, solvent system and reactor type were kept the same for all the films, to understand the effect of surfactant

*For correspondence concentration on the morphology of fabricated FeSe films.

Different methods of chemical vapour deposition (CVD) are preferred based on the type of film required and availability of the precursors. Metallo organic, ${ }^{10}$ low pressure, ${ }^{11}$ atmospheric pressure, ${ }^{12}$ plasma enhanced, ${ }^{13}$ laser-assisted, ${ }^{13}$ liquid injection and molecular beam epitaxy $^{14}$ are the different methods of CVD. These methods require vaporization of the precursors and hence limit the choice of inorganic materials as precursors. So, AACVD was developed which depends on the solubility of the precursor rather than on its vaporization. Another positive aspect of AACVD is its simplified precursor delivery system which nebulizes the precursor solution into small droplets. These droplets (aerosol) are then transferred to the substrate inside a tube furnace with the help of a carrier gas. Conventionally, different elemental powders or their organic compounds are used for the fabrication of binary inorganic materials, but this approach has the disadvantages of impure films, uncontrolled stoichiometry, lower yields due to multiple steps and involvement of hazardous chemicals. These problems can be solved by the use of a single source precursor which has the advantage of good control over stoichiometry and enhanced safety by improved control on temperature, flow and leaks. Single source precursor approach has been adapted for 
the fabrication of oxides, sulfides, ${ }^{15}$ selenides ${ }^{16}$ and tellurides. ${ }^{17}$

Due to the discovery of superconductivity, electrochemical sensing and photocatalytic activity in FeSe films, huge attention has been given to its fabrication by electrodeposition, ${ }^{18}$ pulsed laser deposition, ${ }^{19}$ chemical vapour transport, ${ }^{20}$ chemical bath deposition, ${ }^{21}$ chemical vapour deposition, ${ }^{22}$ spray pyrolysis, ${ }^{23}$ molecular beam epitaxial growth, ${ }^{24}$ solid state reaction ${ }^{25}$ at different sintering temperatures ${ }^{26}$ high temperature solution for thick films ${ }^{27}$ rapid, solvent-less reaction under autogenic pressure at elevated temperature (RAPET), etc. ${ }^{28}$ As reported in this article, we have used AACVD for the morphological studies of FeSe films, fabricated from ferrocene-incorporated selenourea precursor, using SEM, EDS, EDS mapping and PXRD. The mechanism of surfactant action has been determined by scanning the interaction between the $\mathrm{MeP} 2 \mathrm{~F}$ and surfactant with $\mathrm{UV}$-Vis spectroscopy and cyclic voltammetry (CV).

\section{Experimental}

\subsection{Materials and methods}

FeSe thin films were characterized with $\mathrm{CuK} \alpha$ radiation of $0.154 \mathrm{~nm}$ between diffraction angles 10-80 degrees. SEM images were taken on a SEM JEOL model, $5910 \mathrm{LV}$ with an accelerating voltage of $20 \mathrm{kV}$ at high vacuum (HV) mode and secondary electron image (SEI). The semi-quantification elemental analysis, to find out the weight percentages of the elements, were carried out using OXFORD INCA energy dispersive X-ray spectrometer 7274 (EDS). Absorption spectra were recorded on Shimadzu 1800 spectrophotometer. To note the change of absorption, first the spectrum of free $\mathrm{MeP} 2 \mathrm{~F}$ was run and then with different concentrations of triton and span separately. Dilution of all the solutions were kept similar to avoid its effect.

Cyclic voltammetry (CV) was performed on Biologic SP-300 cyclic voltammeter running with EC-Lab Express V 5.40 software, Japan. CV was performed with a setup having three electrodes system i.e., working (platinum disc electrode) reference $(\mathrm{Ag} / \mathrm{AgCl})$ and auxiliary electrodes (platinum wire electrode). Working electrode was polished with alumina powder and rinsed with distilled water before every run. Analytical grade $\mathrm{KCl}$ was used as supporting electrolyte and nitrogen gas $(99.9 \%)$ was used to purge the solution mixture to avoid interference by oxygen. Changes in $\mathrm{CV}$ peak potentials were monitored to obtain information about the mode of interaction. ${ }^{29}$ Values of diffusion coefficients were determined by RandlesSevcik equation: ${ }^{6}$

$$
\mathrm{I}_{\mathrm{pa}}=2.69 \times 10^{5} \mathrm{n}^{3 / 2} \mathrm{~A} \mathrm{C}_{\mathrm{o}}^{*} \mathrm{D}_{\mathrm{o}}^{1 / 2} v^{1 / 2}
$$

where $\mathrm{I}_{\mathrm{pa}}$ is the anodic peak current, $\mathrm{C}_{\mathrm{o}}^{*}$ is the concentration of reductant in $\mathrm{mol} \mathrm{cm} \mathrm{cm}^{-3}, \mathrm{~A}$ is the geometric area of the electrode in $\mathrm{cm}^{2}, \mathrm{n}$ is the number of electrons involved in the process, $D_{0}$ is the diffusion coefficient in $\mathrm{cm}^{2} \mathrm{~s}^{-1} .^{29}$

\subsection{Fabrication of FeSe films by AACVD}

FeSe thin films were deposited using AACVD kit equipped with CARBOLITE furnace (21-101847, type MTS10/15/130) and Deurer living LB44 humidifier, having ultrasonic system on glass substrates. Optimized concentrations of MeP2F (0.08 g) were dissolved in $15 \mathrm{~mL}$ of toluene in a two-necked round bottom flask $(100 \mathrm{~mL})$ equipped with a glass inlet. This inlet was attached to one of the necks to allow the transport of aerosol under the constant flow of argon with varying concentrations of the surfactants (triton and span) for all the fabricated thin films (except film 1 in which no surfactant was used). In this setup, a tube connects the round bottom flask with the reactor tube which was held at $500^{\circ} \mathrm{C}$. The flow rate of argon was controlled with platon flow gauge and kept constant for all the films. Round bottom flask containing the precursor rests on a water bath above piezoelectric modulator of an ultrasonic humidifier. Humidifier generated the aerosol which was moved to hot zone of the reactor where thermally induced reaction generated the FeSe films.

\subsection{Synthesis and characterization of the precursor}

We have recently reported the synthesis and characterization (FT-IR, multi-nuclear NMR, AAS, CHNS and

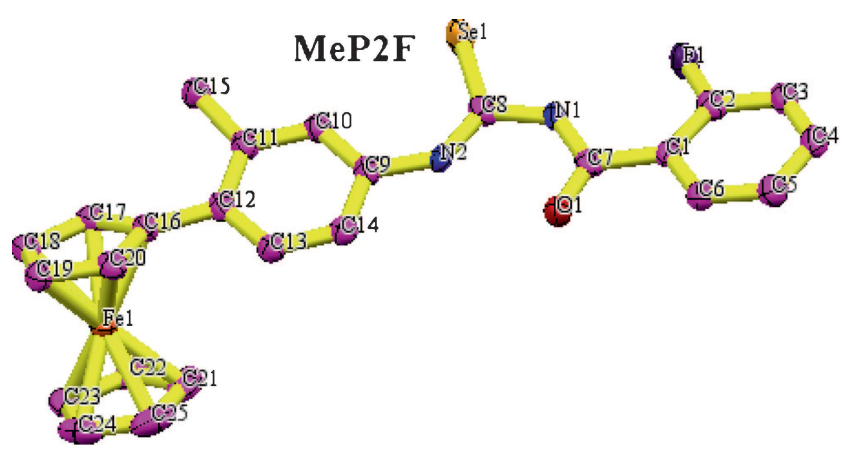

Figure 1. Molecular diagram of MeP2F with ellipsoid displacement. Non-hydrogen atoms are represented by $30 \%$ probability boundary spheres. Hydrogen atoms are not shown for simplicity. ${ }^{6}$ 
single crystal XRD (figure 1 presents the ORTEP of $\mathrm{MeP} 2 \mathrm{~F})$ ) of MeP2F and its biological applications. ${ }^{6}$

\section{Results and Discussion}

Figure 2a represents the TGA (thermogravimetric analysis) of MeP2F, which shows that the decomposition of the precursor starts at $\sim 143^{\circ} \mathrm{C}$ and completes at $478^{\circ} \mathrm{C}$. Keeping in view this information, the temperature during CVD was kept at $500^{\circ} \mathrm{C}$ i.e., a little above the complete decomposition temperature so that the impurity of the carbon is not present in the films. Figure $2 b$ gives the representative EDS mapping images for $\mathrm{Fe}$ and $\mathrm{Se}$ which illustrates the uniform distribution of the elements throughout the film and hence proves the homogeneous nature of the FeSe material deposited. EDS of all the films indicated slight deficiency of the Se with $\mathrm{FeSe}_{\mathrm{x}}(\mathrm{x}=0.81-0.89)$. This is the range which is claimed to be superconducting in previous reports. ${ }^{27,30}$

FeSe generally exhibits two crystalline phases i.e., $\mathrm{PbO}$ type tetragonal phase and NiAs type hexagonal phase. ${ }^{31}$ Recent discovery of superconductivity in tetragonal FeSe has increased the interest in the chemistry of FeSe. ${ }^{32}$ In this study, we observed that our precursor $(\mathrm{MeP} 2 \mathrm{~F})$ provides hexagonal FeSe with maximum intense peak at 32.52 degree $(2 \theta)$ for $(002)$ hexagonal plane when it is subjected to CVD without the use of surfactant (figure 3, XRD pattern 1). Use of the first concentration of the triton $(0.2 \mathrm{~mL}$ of triton $/ 15 \mathrm{~mL}$ of precursor solution) keeping all the other conditions

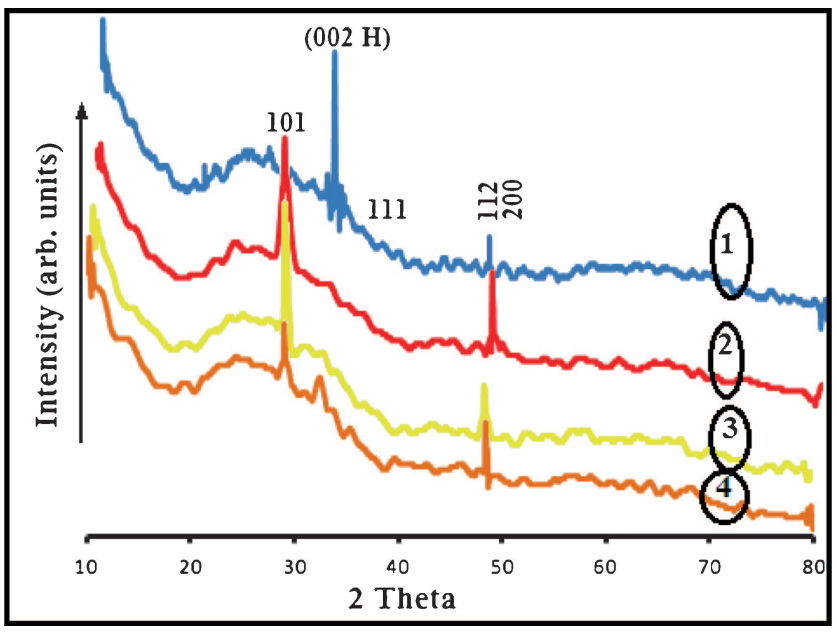

Figure 3. XRD patterns of film 1 (film fabricated from $\mathrm{MeP} 2 \mathrm{~F}$ without using any surfactant during CVD), film 2 (0.2 mL of triton/15 mL of precursor solution), film 3 ( $0.4 \mathrm{~mL}$ of triton $/ 15 \mathrm{~mL}$ of precursor solution) and film 4 (0.6 mL of triton/15 mL of precursor solution).

same, changes the hexagonal phase to tetragonal phase with two prominent peaks; one for (101) plane and another for (112) plane of tetragonal FeSe (figure 3, XRD pattern 2). Second and third concentrations of the triton $(0.4 \mathrm{~mL}$ and $0.6 \mathrm{~mL}$ of triton $/ 15 \mathrm{~mL}$ of precursor solution) keeping all the other conditions same during CVD, yielded tetragonal FeSe as the major phase (figure 3, XRD patterns 3 and 4). Use of span however does not change the phase of the FeSe relative to the phase observed without using surfactant i.e., all the three concentrations of the span $(0.3-0.9 \mathrm{~mL}$ of span/15 $\mathrm{mL}$ of precursor solution) provided hexagonal
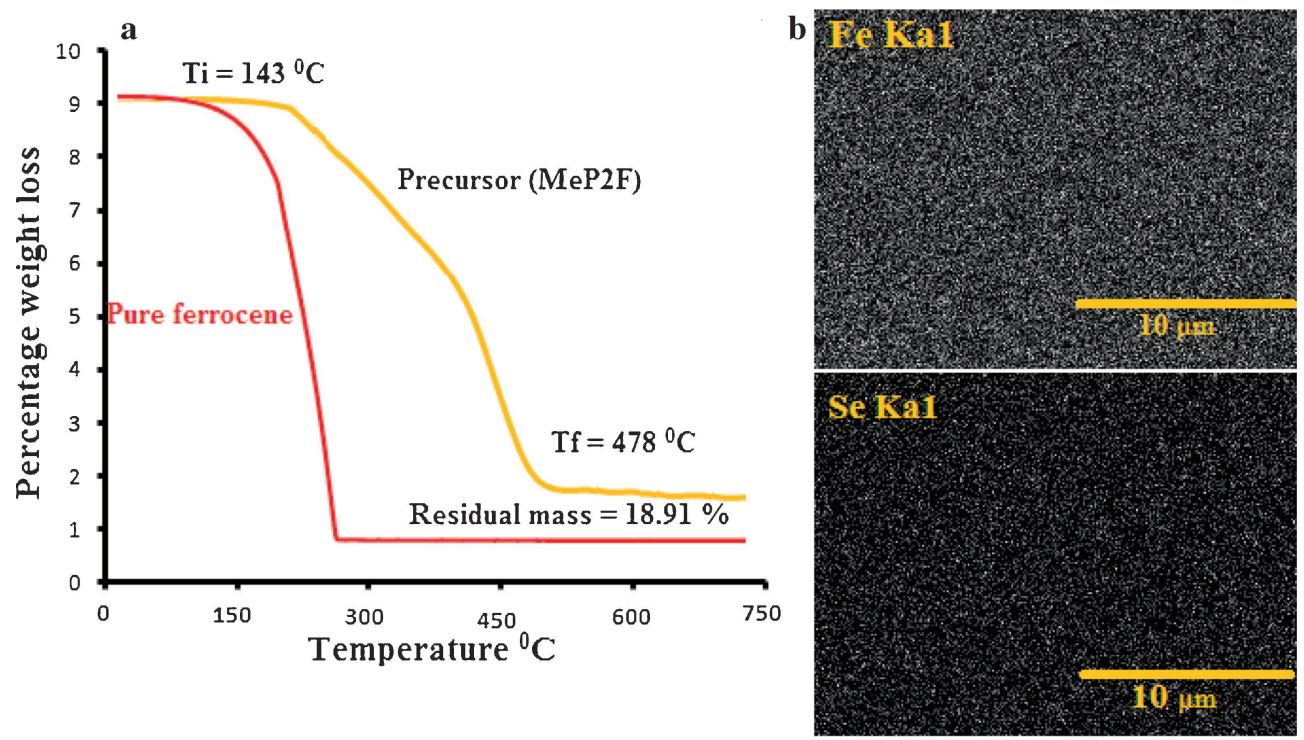

Figure 2. (a) Comparative thermogravimetric analysis of pure ferrocene and MeP2F; (b) Representative EDS mapping images of Fe and Se for film 1 (the film fabricated by AACVD of MeP2F without the use of any surfactant). 


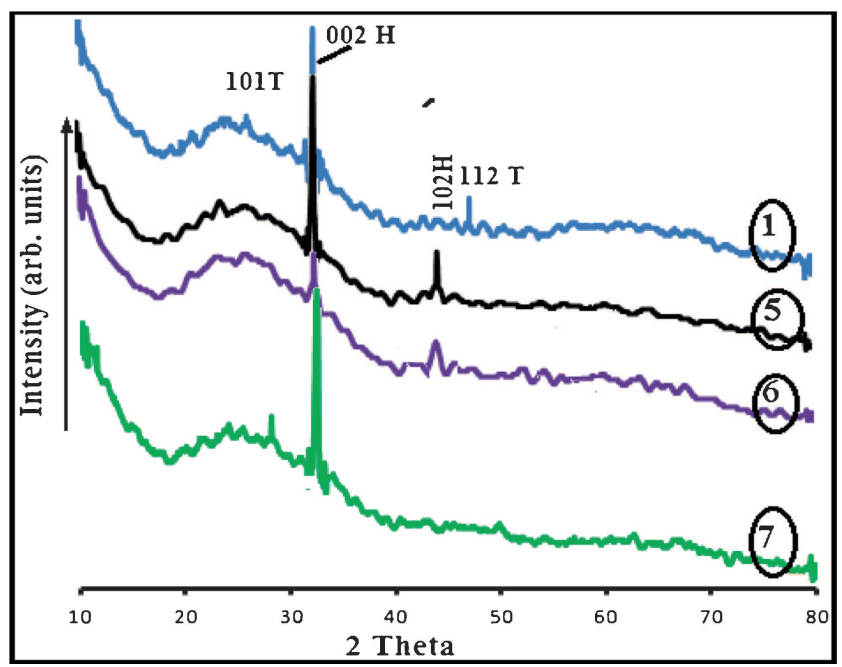

Figure 4. XRD patterns of film 1 (film fabricated from MeP2F without using any surfactant during CVD), film 5 (0.3 $\mathrm{mL}$ of span/15 mL of precursor solution), film $6(0.6 \mathrm{~mL}$ of span/15 mL of precursor solution) and film $7(0.9 \mathrm{~mL}$ of $\mathrm{span} / 15 \mathrm{~mL}$ of precursor solution).

FeSe as the major phase (figure 4, XRD patterns 5-7). The advantage with the use of span however, is that it produces phase-pure material.

Figure 5 displays the comparison between the SEM images of the film produced without the use of a surfactant (film 1) and films produced by using $1^{\text {st }}$
(0.2 $\mathrm{mL}$ of triton $/ 15 \mathrm{~mL}$ of precursor solution), $2^{\text {nd }}$ $\left(0.4 \mathrm{~mL}\right.$ of triton $/ 15 \mathrm{~mL}$ of precursor solution) and $3^{\text {rd }}$ concentration of triton $(0.6 \mathrm{~mL}$ of triton $/ 15 \mathrm{~mL}$ of precursor solution) along with the precursor to fabricate the $\mathrm{FeSe}$ thin films. It can be observed that film 1, generated by AACVD of $\mathrm{MeP} 2 \mathrm{~F}$ is uniform and is adherent to the glass support. Close visualization (at $1 \mu \mathrm{m}$ ) indicates formation of spherical balls of FeSe. By using $1^{\text {st }}$ concentration of triton, separation between the particles increases and modification in the spherical morphology of FeSe is observed (film 2). $2^{\text {nd }}$ concentration of triton (film 3) produces somewhat cubic crystals along with needle like structures whereas $3^{\text {rd }}$ concentration of triton produces well defined cubic crystals of FeSe (film 4).

Effect of span on the morphology of the FeSe films has been elaborated in figure $6.1^{\text {st }}$ concentration of span ( $0.3 \mathrm{~mL}$ of span $/ 15 \mathrm{~mL}$ of precursor solution) decreases the size of the FeSe particles enormously which is evident from the inset image $(1 \mu \mathrm{m})$ in film 5. Doubling the concentration of span $(0.6 \mathrm{~mL}$ of span $/ 1 \mathrm{~mL}$ of precursor solution) produces very fine cubic crystals of FeSe whereas $3^{\text {rd }}$ concentration of the span $(0.9 \mathrm{~mL}$ of span $/ 15 \mathrm{~mL}$ of precursor solution) produces microflowers with petals in the nanometer size. Moreover, topographic and phase scans in the AFM micrographs
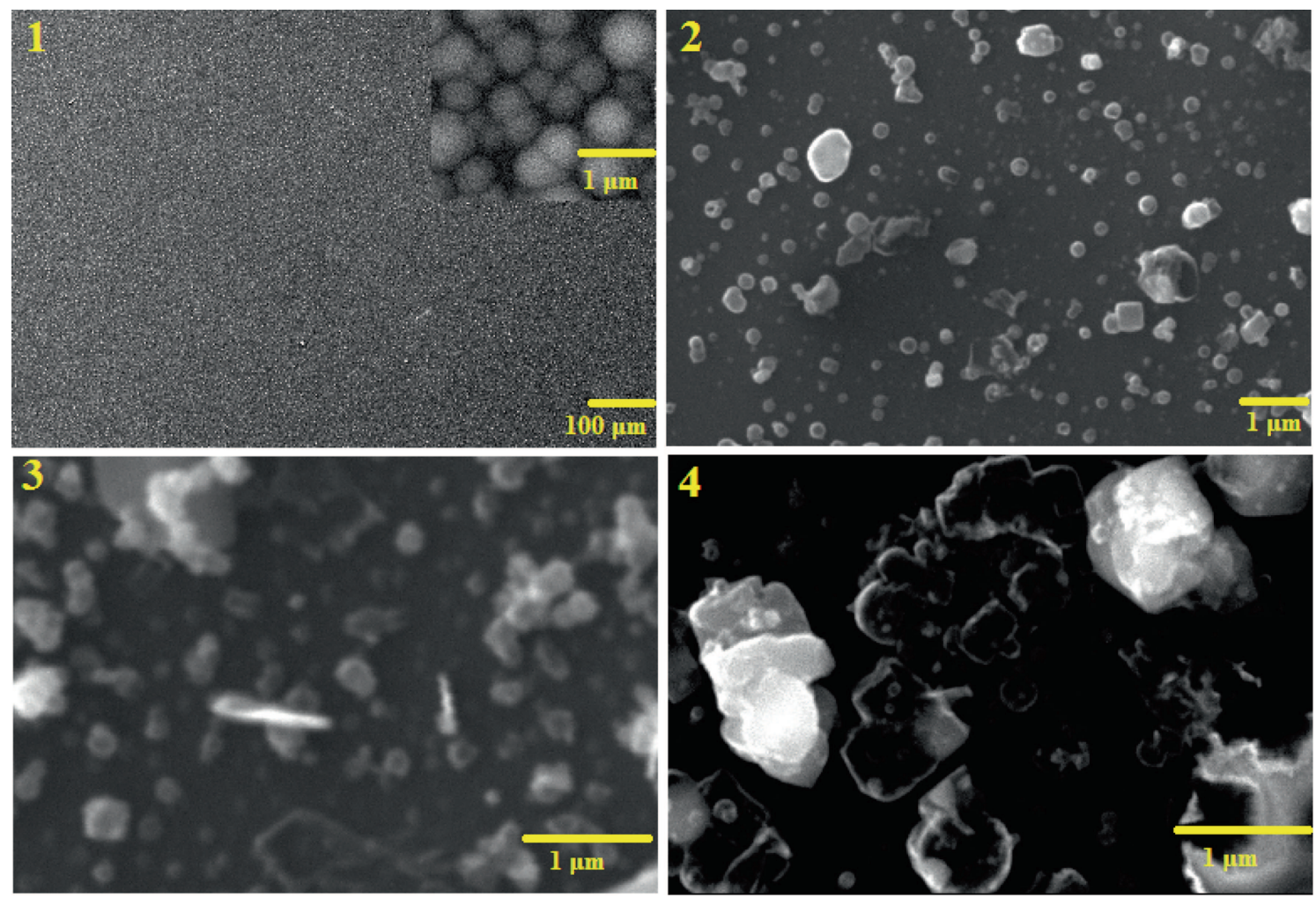

Figure 5. Comparative SEM images of film 1 (film fabricated from MeP2F without using any surfactant during CVD), film 2 (0.2 mL of triton/15 mL of precursor solution), film 3 (0.4 mL of triton/15 mL of precursor solution) and film $4(0.6 \mathrm{~mL}$ of triton/15 $\mathrm{mL}$ of precursor solution). 

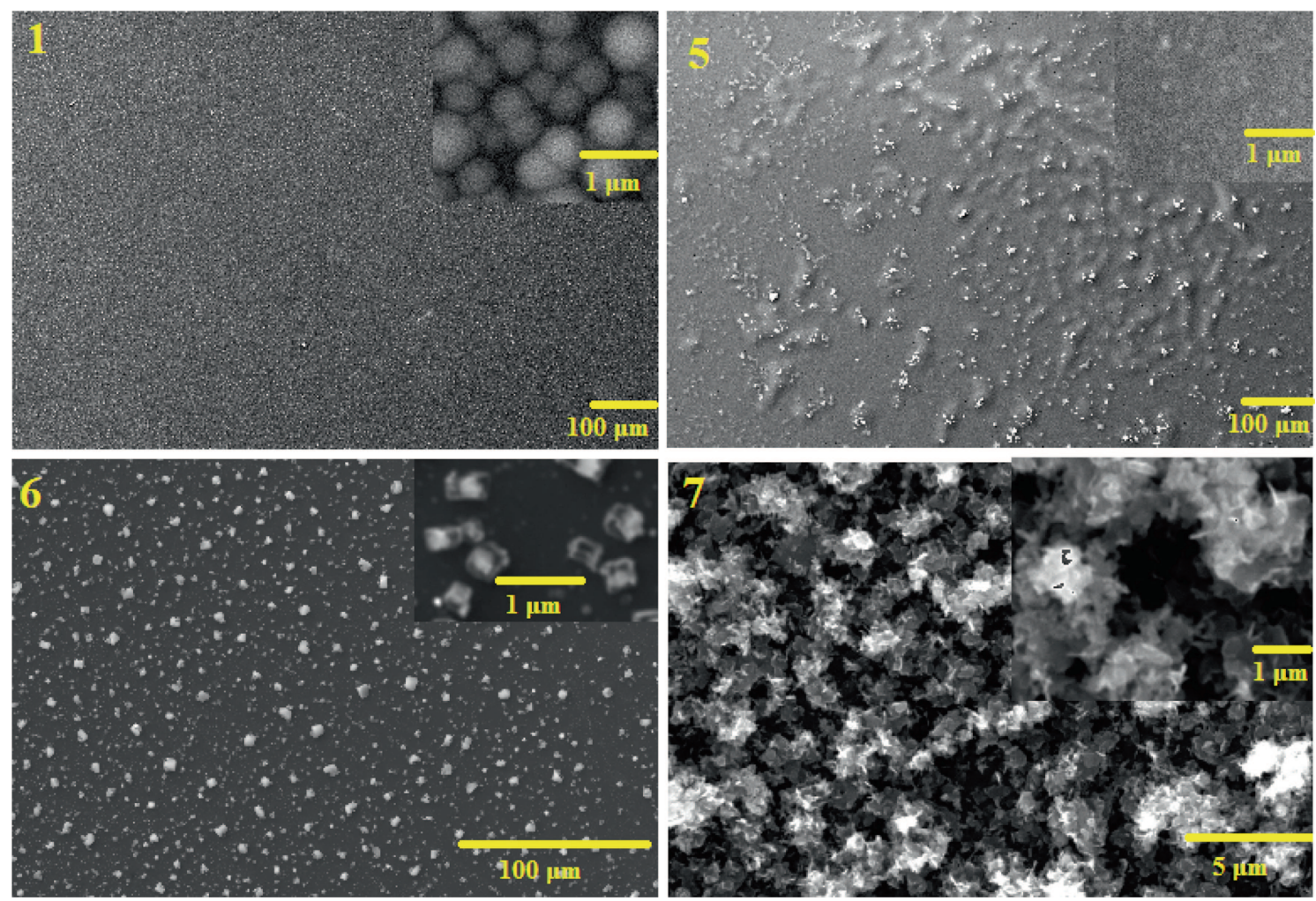

Figure 6. Comparative SEM images of film 1 (film fabricated from MeP2F without using any surfactant during CVD), film 5 (0.3 mL of span/15 mL of precursor solution), film 6 (0.6 mL of span $/ 15 \mathrm{~mL}$ of precursor solution) and film 7 ( $0.9 \mathrm{~mL}$ of span $/ 15 \mathrm{~mL}$ of precursor solution).

of a representative film (film 1) confirm the uniform topography and phase of FeSe throughout the film (figure 7). We propose that this change in morphology of the films is due to the interaction of the surfactants (span and triton) with the precursor (MeP2F) both in the solution phase and in the gas phase. We believe that there is an adduct formation between the surfactant and precursor which is concentration-dependent and governs the change in morphology. We have made an attempt to investigate the mechanism of interaction between the surfactants and MeP2F in the following subheadings.

\subsection{Mechanism for the interaction of surfactants with the precursor}

3.1a UV-Vis spectroscopy: In UV-Vis spectrum, $\mathrm{MeP} 2 \mathrm{~F}$ shows one prominent band at $282 \mathrm{~nm}$ due
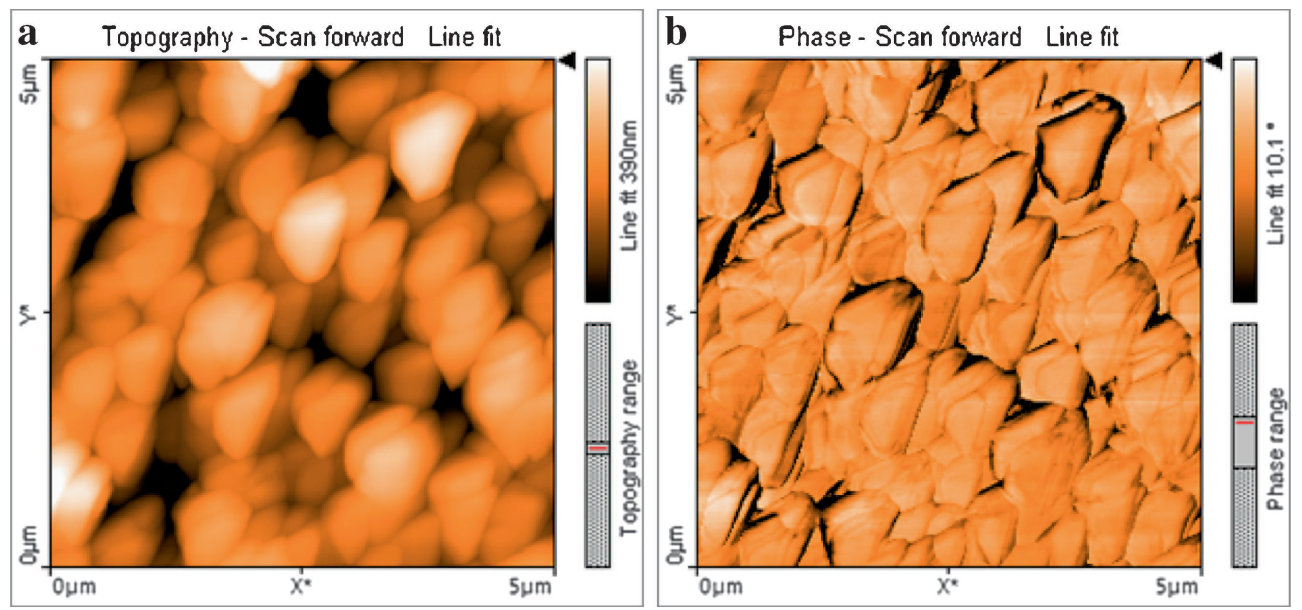

Figure 7. Representative AFM micrographs of film 1(film fabricated from MeP2F without using any surfactant during CVD) (a) Topography scan; (b) Phase scan. 
to 4-ferrocenyl-3-methylphenyl moiety and the broad shoulder between 330 and $360 \mathrm{~nm}$ may be because of the charge transfer between bonding and anti-bonding orbitals of $\mathrm{Cp}$ (cyclopentadienyl) ring and iron atom. ${ }^{7}$ In an attempt to determine the mode of interaction between the MeP2F and triton, increasing concentration of triton were used with fixed concentration of MeP2F (by keeping the dilution constant). Hypochromism with red shift $(7 \mathrm{~nm})$ of triton-MeP2F adduct relative to the free $\mathrm{MeP} 2 \mathrm{~F}$ indicated the ease of the corresponding transition in triton-MeP2F adduct. Triton-MeP2F binding constant $(\mathrm{K})$ was determined by a plot of A0/A-A0 vs. $1 /$ [triton] according to Eq. $2^{33}$ with a value equal to $1.08 \times 10^{2} \mathrm{M}^{-1}$ (figure 8).

$$
\frac{\mathrm{A}_{\mathrm{o}}}{\mathrm{A}-\mathrm{A}_{\mathrm{o}}}=\frac{\varepsilon_{\mathrm{F}}}{\varepsilon_{\mathrm{C}-\mathrm{F}}-\varepsilon_{\mathrm{F}}}+\frac{\varepsilon_{\mathrm{F}}}{\varepsilon_{\mathrm{C}-\mathrm{F}}-\varepsilon_{\mathrm{F}}} \cdot \frac{1}{\mathrm{~K}[\text { Surfactant }]}
$$

where, $\mathrm{K}$ is the binding constant, $\mathrm{A}_{\mathrm{o}}$ is the absorbance of free MeP2F (F), A is the absorbance of $\mathrm{MeP} 2 \mathrm{~F}$-surfactant adduct $(\mathrm{C}-\mathrm{F}), \varepsilon_{\mathrm{F}}$ and $\varepsilon_{\mathrm{C}-\mathrm{F}}$ are the absorption coefficients of $\mathrm{F}$ and $\mathrm{C}-\mathrm{F}$, related with $\mathrm{A}_{\mathrm{o}}$ and $\mathrm{A}$, respectively. $\mathrm{K}$ is obtained as the ratio of intercept to slope in the plot of $\mathrm{A}_{0} / \mathrm{A}-\mathrm{A}_{0}$ vs $1 /$ [surfactant].

However, during the interaction between span and MeP2F, hyperchromism and blue shift of $11 \mathrm{~nm}$ was observed. This indicates the electrostatic interaction between the electronegative head of the surfactant with positively charged iron atom of the ferrocene. Such electrostatic interaction was not possible for triton and $\mathrm{MeP} 2 \mathrm{~F}$ because electron rich head was absent in the molecular structure of triton. Span-MeP2F interaction constant $(\mathrm{K})$ is equal to $3.15 \times 10^{2} \mathrm{M}^{-1}$ which shows that interaction between span and MeP2F is stronger than the interaction between triton and $\mathrm{MeP} 2 \mathrm{~F}$ (figure 8).

3.1b Cyclic voltammetry: Mode and magnitude of the interaction was further confirmed with $\mathrm{CV}$ as well. In $\mathrm{CV}, \mathrm{MeP} 2 \mathrm{~F}$ gives a couple of well-defined redox peaks in a $100 \%$ reversible electrochemical
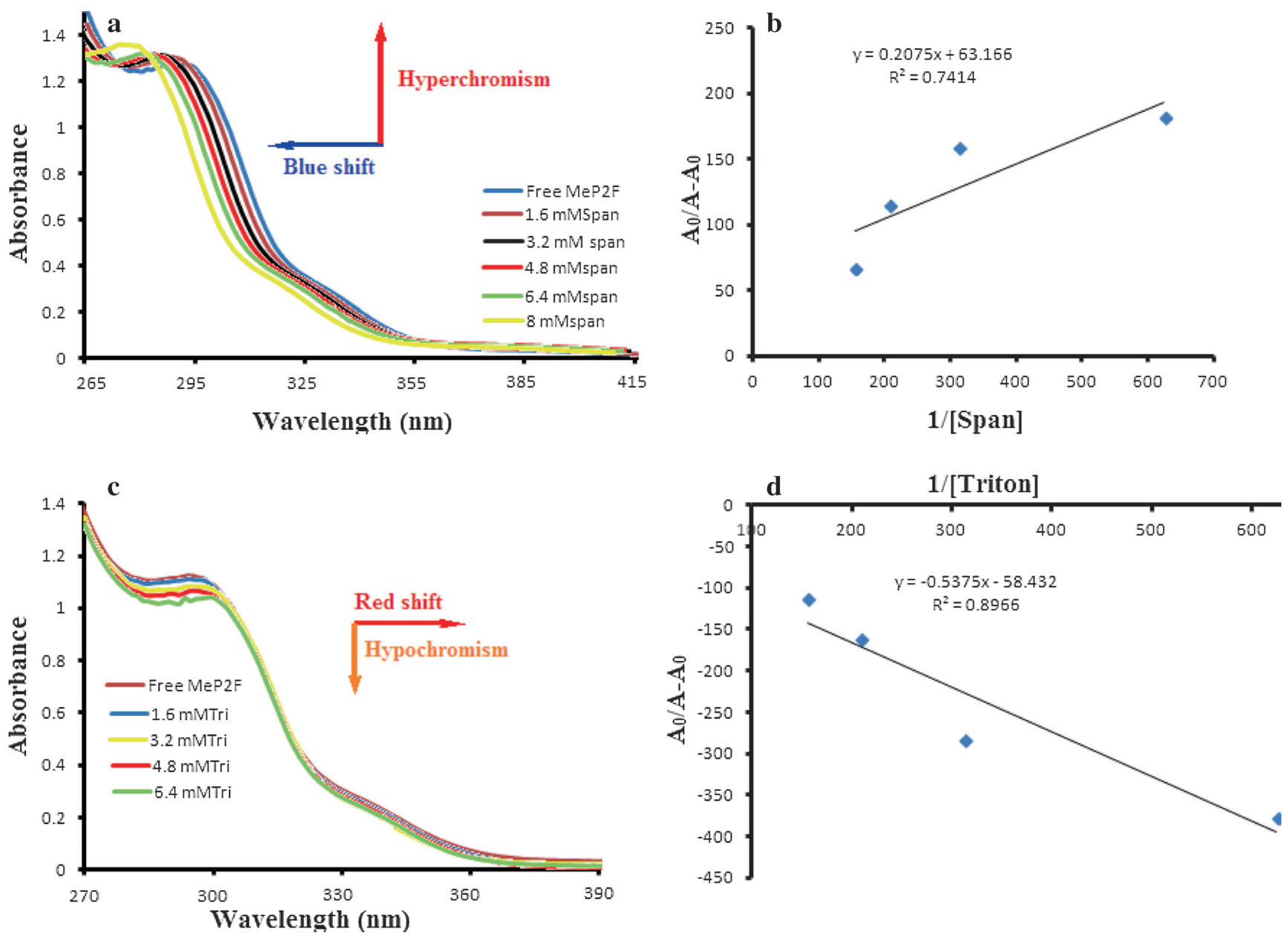

Figure 8. (a) UV-Vis spectra for MeP2F for different concentrations of span in ethanol; (b) Plot of A0/A-A0 vs. 1/[Span] $\left(\mathrm{mol} \mathrm{dm}^{-3}\right)$, see eq. 2 ; (c) UV-Vis spectra for MeP2F for different concentrations of triton in ethanol; (d) Plot of A0/A-A0 vs. $1 /\left[\right.$ Triton] $\left(\mathrm{mol} \mathrm{dm}^{-3}\right)$, see eq. 2 . 

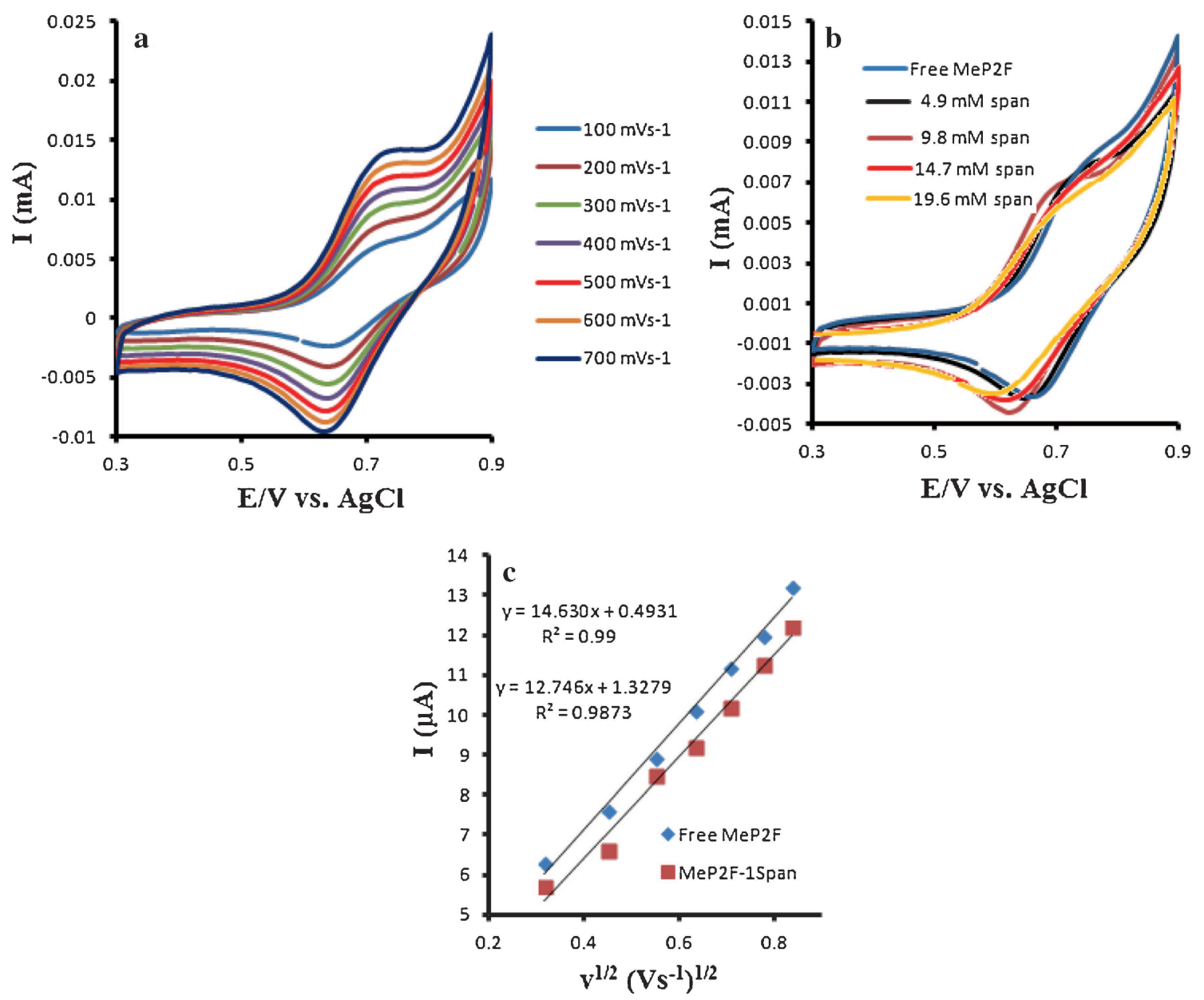

Figure 9. (a) Plots of $\mathrm{I}(\mathrm{mA})$ vs. $\mathrm{E}(\mathrm{V})$ at different scan rates for MeP2F showing $100 \%$ reversible electrochemical process. (b) Comparative cyclic voltammograms for free MeP2F and span-MeP2F adducts showing a decrease in current with negative shift in the peak potential. (c) Plots of I $(\mu \mathrm{A})$ vs. $v^{1 / 2}$ for the determination of diffusion coefficients.

process (figure 9a). The reversibility of the process was determined by following three parameters: ${ }^{29}$ Constant voltage of oxidation and reduction peaks at different scan rates, almost equal currents of the oxidation and reduction peaks and a difference of $\sim 59 \mathrm{mV}$ between the oxidation and reduction peaks. When concentration of the span was varied for fixed concentration of MeP2F (dilution was kept constant by using different CV cell in each addition), a negative shift (7, 23, 31 and $39 \mathrm{mV}$ for four concentrations of span, see figure $9 \mathrm{~b}$ ) in the peak potential was observed with a decrease in the current (figure 9b). This negative shift in the peak potential indicates the electrostatic mode of interaction between the positively charged $\mathrm{Fe}$ atom of the ferrocene and electronegative head of the span. This negative shift occurs due to the easy oxidation of MeP2F-span adducts relative to free $\mathrm{MeP} 2 \mathrm{~F}$ when electrons become available to it by the electrostatic interaction between the electronegative head of span with positively charged ferrocenium moiety. Formation of adduct between the span and MeP2F was confirmed by measuring the diffusion coefficients of the free MeP2F and span-MeP2F adduct according to Eq. 2. Lower diffusion coefficient of span-MeP2F $\left(4.45 \times 10^{-7} \mathrm{~cm}^{2} \mathrm{~s}^{-1}\right)$ than free MeP2F $\left(5.87 \times 10^{-7} \mathrm{~cm}^{2} \mathrm{~s}^{-1}\right)$ indicates the slow approach of adduct to the electrode due to its higher molecular mass (figure 9c). Whereas in case of triton-MeP2F a positive shift in the potential of triton-MeP2F was observed relative to free $\mathrm{MeP} 2 \mathrm{~F}$ which eliminates any chance of electrostatic interaction. The diffusion coefficient of triton-MeP2F $\left(5.23 \times 10^{-7} \mathrm{~cm}^{2} \mathrm{~s}^{-1}\right)$ was also found to be lower than the free MeP2F $\left(5.87 \times 10^{-7} \mathrm{~cm}^{2} \mathrm{~s}^{-1}\right)$ (figure 10).

3.1c Electrical conductivity measurements: For electrical properties of $\mathrm{FeSe}$, a representative film was coated on ITO conducting glass by AACVD using $\mathrm{MeP} 2 \mathrm{~F}$. Electrical conductivity of this film was determined by two-probe DC conductivity method between 

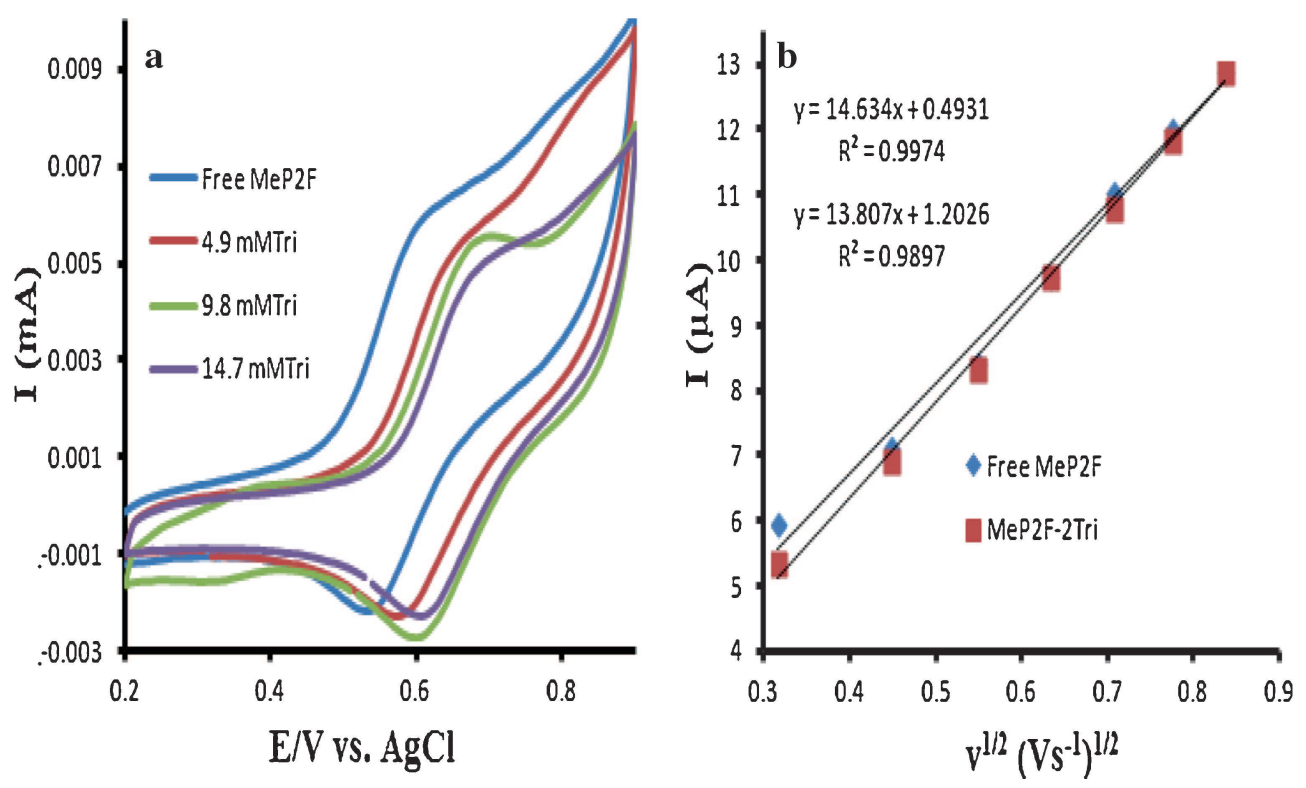

Figure 10. (a) Comparative cyclic voltammograms for free $\mathrm{MeP} 2 \mathrm{~F}$ and triton-MeP2F adducts showing a decrease in current with positive shift in the peak potential. (b) Plots of I $(\mu \mathrm{A})$ vs. $v^{1 / 2}$ for the determination of diffusion coefficients.

the temperature ranges of 298.15 to $389.15 \mathrm{~K}$ by Digital Multimeter Model DMM 2001, Laboratory Power supply 30V/5A, Laboratory Oven. For the measurement of electrical resistivity the formula used is,

$$
\rho=R A / L,
$$

where $\rho$ is electrical resistivity, $\mathrm{R}$ is electrical resistance, $\mathrm{A}$ is cross sectional area in $\mathrm{cm}^{2}$ and $\mathrm{L}$ is length in $\mathrm{cm}$.

Figure 11 presents the plot of electrical resistivity vs. I/T of a representative FeSe thin film coated on

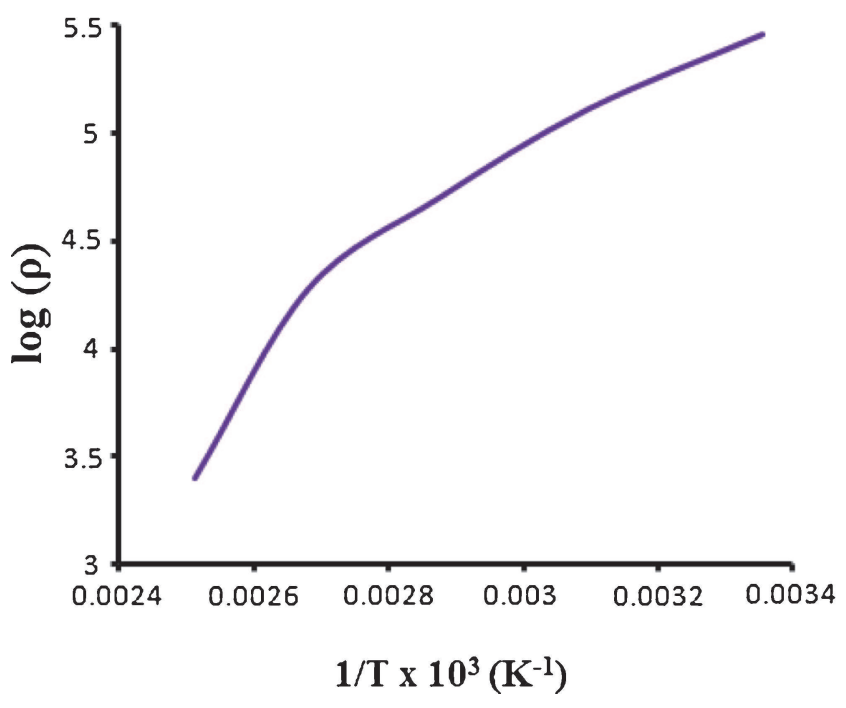

Figure 11. Representative temperature dependence of the electrical resistivity, $\rho$ of a FeSe film coated on ITO glass using MeP2F alone without using any surfactants.
ITO glass fabricated by using single source precursor (MeP2F) with the help of AACVD. Electrical resistivity was measured at five different temperatures i.e., $298.15,323.15,348.15,373.15$ and $398.15 \mathrm{~K}$ and corresponding resistivity values were, $29 \times 10^{4}, 13 \times$ $10^{4}, 5.0 \times 10^{4}, 1.9 \times 10^{4}$ and $0.25 \times 10^{4} \mathrm{Ohm}-\mathrm{cm}$, respectively.

\section{Conclusions}

In this article, we have described and discussed successful convertion of ferrocene-incorporated selenourea precursor (MeP2F) to FeSe thin films with the help of aerosol-assisted chemical vapour deposition (AACVD). We have been able to present the effect of different concentration of triton and span (surfactants) on the crystalline phases and morphologies of the films produced by PXRD and SEM. We have also provided a glimpse of the interaction between the precursor and surfactants with the help of UV-Vis spectroscopy and cyclic voltammetry which governs the phase and morphology changes.

\section{References}

1. John V T, Simmons B, McPherson G L and Bose A 2002 Curr. Opin. Colloid Interface Sci. 7 288

2. Praveen kumar C M, Venkatesha T V and Chandrappa K G 2012 Surf. Coat. Technol. 2062249

3. Zhou X and Wang X 2013 Optik 1241038 
4. Guo X, Yi P, Yang Y, Cui J, Xiao S and Wang W 2013 Electrochimica Acta. 90524

5. McNally C S, Turner D P, Kulak A N, Meldrum F C and Hyett G 2012 Chem. Commun. 481490

6. Hussain R A, Badshash A, Sohail M, Lal B and Altaf A A 2013 Inorg. Chim Acta. 402133

7. Hussain R A, Badshah A, Sohail M, Lal B and Akbar K 2013 J. Mol. Struct. 1048367

8. Hussain R A, Badshah A, Tahir M N, Hassan Tu and Bano A 2013 J. Biochem. Mol. Toxicol. 2860

9. Hussain R A, Badshah A, Yasmin F, Khan M D and Tahir N M 2015 Aust. J. Chem. 68298

10. Moniz S J, Blackman C S, Carmalt C J and Hyett G 2010 J. Mater. Chem. 207881

11. Blackman C S, Carmalt C J and Parkin I P et al. 2002 Dalton Trans. 132702

12. Thomas T, Blackman C S, Parkin I P and Carmalt C J 2010 Eur. J. Inorg. Chem. 365629

13. Choy K L 2003 Prog. Mater Sci. 4857

14. Marchand P, Hassan I A, Parkin I P and Carmalt C J 2013 Dalton Trans.

15. Ashraf S, Saeed A and Malik M A et al. 2013 Eur. J. Inorg. Chem.

16. Akhtar J, Akhtar M, Malik M A, O'Brien P and Raftery J 2012 J. Am. Chem Soc. 1342485

17. Berlincourt D, Jaffe H and Shiozawa L 963 Phys. Rev. 11291009

18. Demura S, Okazaki H and Ozaki T et al. 2013 Solid State Commun. 15440
19. Chen T-K, Luo J-Y and Ke C-T et al. 2010 Thin Solid Films. 5191540

20. Hara Y, Takase K and Yamasaki A et al. 2010 Physica C: Superconductivity. 470 Supplement 1:S313

21. Ubale A, Sakhare Y and Bombatkar S 2013 Mater. Res. Bull.

22. Wu X J, Zhang Z Z and Zhang J Y et al. 2008 Thin Solid Films. 5166116

23. Ubale A and Sakhare Y 2013 J. Phys. Chem. Solids.

24. Agatsuma S, Yamagishi T, Takeda S and Naito M 2010 Physica C: Superconductivity. 4701468

25. Li Z, Ju J, Tang J, Sato K, Watahiki M and Tanigaki K 2010 J. Phys. Chem. Solids. 71(4) 495

26. Umeyama $\mathrm{N}$, Tomura $\mathrm{M}$ and Takase $\mathrm{K}$ et al. 2010 Physica C: Superconductivity. 470 Supplement 1:S518

27. Qi X, Wang J-Y, Kuo J-C, Yates K A, Cohen L F 2011 J. Alloys Comp. 509(22) 6350

28. Chang C-C, Wang C-H, Wen M-H, Wu Y-R, Hsieh Y-T and Wu M-K 2012 Solid State Commun. 152649

29. Hussain R A, Badshah A, Tahir M N, Lal B and Khan I A 2013 Aust. J. Chem. 66626

30. Margadonna S, Takabayashi Y and McDonald M T et al. 2008 Chem. Commun. 435607

31. Sines I T and Schaak R E 2010 J. Am. Chem Soc. 133(5) 1294

32. Mishra S, Song K, Koza J A and Nath M 2013 ACS nano. 71145

33. Weiss J 1952 J. Am. Chem. Soc. 74811 